children below 5 years. Would FIMNCI training impart the confidence and skills required for implementation of this training were our research questions.

Methods Cross-sectional Questionnaire based survey from 53 participants of three FIMNCI trainings. $\mathrm{MOs}$ and $\mathrm{SNs}$ belonged the Primary Health Centres (PHCs), Community health Centres (CHCs) and Civil hospitals. Questions related to availability of facilities at their working place; confidence in ability to perform skill based procedures and acceptability and implementation of FIMNCI.

Results The PHCs and CHCs have adequate facilities to treat non critical problems but most lack facilities for intensive care. Most $\mathrm{MOs}(84.2 \%)$ and SNs $(79.4 \%)$ are confident of triage in emergency room as well as providing positive pressure ventilation. All MOs and most SNs (61.9\%) were confident in treating sick children at CHCs while most MOs (66.6\%) and SNs (83.3\%) were not confident at PHCs. Most participants preferred that FIMNCI training should be of longer duration. SNs preferred training in local language. Most MOs were not confident in monitoring of sick children.

Conclusion More focused training should be provided for the staff of PHCs and CHCs like Triage and Resuscitation. Advanced care for various serious illnesses in children cannot be imparted by short training courses.

\section{IMPROVING OUALITY OF CARE FOR HEALTHY TERM JAUNDICED BABIES: COMMUNITY BASED INTEGRATED CARE PATHWAY REDUCE READMISSIONS AND LENGTH OF STAY}

doi:10.1136/archdischild-2012-302724.1734

'D Thakkar, 'S Davies, 'N Callender, ${ }^{2} \mathrm{~S}$ Chalkley, ${ }^{2} \mathrm{~K}$ Phekoo, ${ }^{1} \mathrm{JO}$ Menakaya. ${ }^{1}$ Neonatal Paediatrics, Hillingdon Hospital NHS Foundation Trust; ${ }^{2}$ Imperial College, London, UK

Background Readmission rates for healthy term neonates with severe hyperbilirubinaemia has increased in recent years. In 2011, we implemented a comprehensive risk assessment based integrated care pathway situated in the community to monitor all jaundiced babies. The pathway comprises intensive feeding support; monitoring bilirubin levels at home with transcutaneous bilirubinometers (TcB) and total serum bilirubin (TsB); prompt referral to hospital when thresholds for treatment set at $340 \mu \mathrm{mol} / 1$ was reached and a standardised weaning strategy for phototherapy dosage.

Aims To evaluate the impact of this pathway on variations in decision to treat, readmission rates for jaundiced babies and length of stay (LoS) before and after implementation.

Methods We analysed the case records and compared the outcomes for all healthy term babies who were readmitted to receive phototherapy between 1 June and 30 September 2010 with those of babies admitted during the same period in 2011. We used SPSS software for statistical analysis.

Results 2921 term babies were delivered during the two time epochs. 28/1468 (0.02\%) received phototherapy in 2010 compared with $19 / 1453(0.013 \%)$ in 2011 . The mean maximum bilirubin levels in 2010 was significantly lower at $292 \pm 64 \mu \mathrm{mol} / \mathrm{l}$ (range: 193-457) compared with $362+26.3 \mu \mathrm{mol} / 1$ (range: 323-433) in 2011. The LoS was significantly reduced at $45.5 \pm 26.7 \mathrm{hr}$ in 2011 compared with $87.2 \pm 53.8 \mathrm{hr}$ in 2010 .

Conclusions This study showed reduced readmission rate and a statistically significant reduction in the length of stay during readmission in the post intervention group despite a significantly increased maximum bilirubin level. In addition, there was improved consistency amongst professionals on when to refer babies for phototherapy.

\section{RESULTS OF CHILDREN AND ADULTS ALLERGY TESTING USING THE FAST ALLERGY TEST (FAST CHEK POC)}

doi:10.1136/archdischild-2012-302724.1735
'E Karabeg, 'E Karabeg, ${ }^{2}$ A Karabeg. ' Joint Praxis 'Dr Karabeg', Sanski Most, BosniaHerzegovina; ${ }^{2}$ School of Medicine, Medical University, Maribor, Slovenia

Purpose Analyse the results of allergy testing based on age and gender of patients, frequency of the testing:the inhalation- and nutritional allergy in vitro tests.

Methods 230 persons: 141 (60.87\%) - children, adults $89(38.69 \%)$; from 1 to 70 years old, 108 - males (46.95\%) and 122 females (53.04\%).

Results All patients had some of allergy clinical symptoms: allergic rhinitis, asthma, allergic dermatitis ...

Testing the adults: females $69.66 \%$, males $30.33 \%$; the children girls $42.55 \%$, boys $57.44 \%$.

The inhalation allergy: in children $-82.26 \%$, in adults $72.22 \%$ while the nutritive allergy was in children $17.73 \%$ and $27.77 \%$ in adults.

Inhalation allergy: male children $48.22 \%$, adults $23.59 \%$, female children $34.04 \%$, adults $50.56 \%$.

Nutritional allergy: male children $9.21 \%$, adults $7.86 \%$, female children $8.51 \%$, adults $20.22 \%$.

Frequency of the inhalation allergy: male $48.90 \%$ and female $51.09 \%$; and the nutritional allergy: male $40 \%$, female $60 \%$.

The children: Ambrosija 40.42\%, Alternaria 36.17\%, house dust $31.91 \%$, Cladosporium $21.98 \%$, Hasel pollen $19.14 \%$;

In adults: house dust $39.32 \%$, Ambrozija $34.83 \%$, Alternaria $31.46 \%$, Cladosporium $22.47 \%$, Hasel pollen $21.34 \%$.

Nutritive reagents in children: fish $4.25 \%$, carrot $3.54 \%$, celery $3.54 \%$, wheat flour 2.83 and in adults wheat flour $8.98 \%$, meat $7.86 \%$, fish $4.49 \%$, soybean $4.49 \%$.

Conclusion It was significantly greater need for conducting of allergy tests in children then in adults (61.3\% versus $38.69 \%)$. The inhalation alergenes $78.69 \%$ - nutritive alergenes $21.31 \%$.

The nutritive allergies e often in female population.

\section{6 EFFECT OF EARLY INTERVENTION IN DOWN SYNDROME: A PILOT STUDY IN YOUNG INFANTS}

doi:10.1136/archdischild-2012-302724.1736

${ }^{1} \mathrm{~S}$ Bargagna, ${ }^{2} \mathrm{M}$ Bozza, ${ }^{2} \mathrm{G}$ Purpura, ${ }^{1} \mathrm{~T}$ Luongo. ${ }^{'}$ Child Neurology and Psychiatry; ${ }^{2}$ Stella Maris Institute, Pisa, Italy

Background and Aims Down Syndrome (DS) is the most important genetic cause of intellectual disability. No consensus exists on the specific type and timing of early intervention in this population. Studies on animal models support the beneficial effects of environmental enrichment (EE). Infant Massage (IM) is a good human paradigm of EE. We aim to determine the effects of early EE in young infants with DS.

Methods 12 subjects were randomized in two intervention groups: GroupA received a bi-monthly counseling intervention and includes 5 infants- GroupB in addition to the counseling intervention, the infants received IM performed by a parents; includes 7 infants. A structured family counseling consisting of observation of parentchild interaction in emotional aspects and developmental skills. The study -group B receive an additional intervention based on IM.

Main Outcome Measures Dubowitz Infant Neurological assessment, Prechtl's method; Teller acuity cards, Frisby Stereotest; Parent to Infant Attachment Scale, Parental Stress Index; Griffiths Developmental Mental Scales.

Preliminary Results Motor milestones: upright posture at GroupB 11.3 months, GroupA 12.5 months, crawling GroupB 11.7 months, GroupA 13.5 months; coastal shipping GroupB 14.5 months, GroupA 15 months.

Cognitive level GroupB (OS Total 75.3 GroupA (OS Total 71.5); stereopsis in GroupB (5 months) GroupA (6 months) visual acuity in GroupB (12.5 cy/cm at 12 months) GroupA (10.3 cy/cm at 12 months). 